\title{
On the notion of gauge symmetries of generic Lagrangian field theory
}

G.GiachettA ${ }^{1}$

Department of Mathematics and Informatics, University of Camerino, 62032 Camerino $(\mathrm{MC})$, Italy

L.MANGIAROTTI ${ }^{2}$

Department of Mathematics and Informatics, University of Camerino, 62032 Camerino $(\mathrm{MC})$, Italy

G. SARDANASHVILY ${ }^{3}$

Department of Theoretical Physics, Moscow State University, 117234 Moscow, Russia

General Lagrangian theory of even and odd fields on an arbitrary smooth manifold is considered. Its non-trivial reducible gauge symmetries and their algebra are defined in this very general setting by means of the inverse second Noether theorem. In contrast with gauge symmetries, non-trivial Noether and higher-stage Noether identities of Lagrangian theory can be intrinsically defined by constructing the exact Koszul-Tate complex. The inverse second Noether theorem that we prove associates to this complex the cochain sequence with the ascent operator whose components define non-trivial gauge and higher-stage gauge symmetries. These gauge symmetries are said to be algebraically closed if the ascent operator can be extended to a nilpotent operator. The necessary conditions for this extension are stated. The characteristic examples of Yang-Mills supergauge theory, topological ChernSimons theory, gauge gravitation theory and topological BF theory are presented.

\section{INTRODUCTION}

Treating gauge symmetries of Lagrangian field theory, one is traditionally based on an example of the Yang-Mills gauge theory of principal connections on a principal bundle $P \rightarrow X$ with a structure Lie group $G$. In this theory, gauge transformations are defined as vertical automorphisms of $P$. Infinitesimal generators of one-parameter groups of these

\footnotetext{
${ }^{1}$ Electronic mail: giovanni.giachetta@unicam.it

${ }^{2}$ Electronic mail: luigi.mangiarotti@unicam.it

${ }^{3}$ Electronic mail: sardanashvi@phys.msu.ru
} 
automorphisms are $G$-invariant vertical vector fields on $P$. They are identified with global sections $\xi$ of the quotient $V P / G$ of the vertical tangent bundle $V P$ of $P \rightarrow X$ with respect to the right action of $G$ on $P$. These sections take a local form $\xi=\xi^{p}(x) e_{p}$ where $\left\{e_{p}\right\}$ is the basis for the Lie algebra $\mathfrak{g}$ of $G$. They constitute a Lie $C^{\infty}(X)$-algebra $\mathfrak{g}(X)$ with respect to the bracket

$$
[\xi, \eta]=c_{p q}^{r} \xi^{p} \eta^{q} e_{r}
$$

where $c_{p q}^{r}$ are structure constants of $\mathfrak{g}$. Being $G$-equivariant, principal connections on a principal bundle $P$ are represented by global sections of the quotient

$$
C=J^{1} P / G
$$

of the jet bundle $J^{1} P$ of $P$ which is coordinated by $\left(x^{\mu}, a_{\mu}^{r}\right) .{ }^{1}$ Vertical automorphisms of $P$ yield automorphisms of the bundle $C(2)$. Infinitesimal generators of one-parameter groups of these automorphisms are given by vector fields

$$
u_{\xi}=\left(\partial_{\lambda} \xi^{r}+c_{p q}^{r} a_{\lambda}^{p} \xi^{q}\right) \frac{\partial}{\partial a_{\lambda}^{r}}
$$

on $C$. They form a real Lie algebra

$$
\begin{aligned}
& u_{\xi}+u_{\eta}=u_{\xi+\eta}, \quad \lambda u_{\xi}=u_{\lambda \xi}, \quad \lambda \in \mathbb{R}, \\
& {\left[u_{\xi}, u_{\eta}\right]=u_{[\xi, \eta]},}
\end{aligned}
$$

which is isomorphic to the Lie algebra (1) seen as a real algebra (but not the $C^{\infty}(X)$-one because $\left.u_{f \xi} \neq f u_{\xi}, f \in C^{\infty}(X)\right)$. This isomorphism is a linear differential operator on sections of $V P / G \rightarrow X$. The vector fields (3) are exact symmetries of the Yang-Mills Lagrangian $L_{Y M}$, i.e., the Lie derivative of $L_{Y M}$ along any $u_{\xi}(3)$ vanishes. They are called the gauge symmetries of $L_{Y M}$ depending on gauge parameters $\xi \in \mathfrak{g}(X)$.

This notion of gauge symmetries has been generalized to Lagrangian field theory on any fiber bundle $Y \rightarrow X$ over an $n$-dimensional smooth manifold $X$ as follows. ${ }^{3,4}$ Given a $k$-order jet manifold $J^{k} Y$ of $Y$, let us consider the pull-back

$$
T^{k} Y=T Y \underset{Y}{\times} J^{k} Y
$$

of the tangent bundle $T Y$ of $Y$ onto $J^{k} Y$ over $Y$. Sections of $T^{k} Y \rightarrow J^{k} Y$ are called generalized vector fields on $Y .^{2}$ A generalized vector field $u$ is said to be a variational symmetry of a Lagrangian $L$ if the Lie derivative of $L$ along $u$ is a variationally trivial 
Lagrangian. Variational symmetries constitute a real subspace $\mathcal{G}_{L}$ of the $C^{\infty}\left(J^{k} Y\right)$-module of generalized vector fields.

Definition 1: Let $E \rightarrow X$ be a vector bundle and $E(X)$ the $C^{\infty}(X)$ module $E(X)$ of sections of $E \rightarrow X$. Let $\zeta$ be a linear differential operator on $E(X)$ taking values in the space $\mathcal{G}_{L}$ of variational symmetries. Elements $u_{\phi}=\zeta(\phi)$ of $\operatorname{Im} \zeta$ are called gauge symmetries of a Lagrangian $L$ parameterized by sections $\phi$ of $E \rightarrow X$.

Equivalently, these gauge symmetries are given by a section $\widetilde{\zeta}$ of a fiber bundle

$$
T Y \underset{Y}{\times} J^{k} Y \underset{Y}{\times}\left(J^{m} E \underset{X}{\times} Y\right) \rightarrow J^{k} Y \underset{Y}{\times}\left(J^{m} E \underset{X}{\times} Y\right)
$$

such that $u_{\phi}=\zeta(\phi)=\widetilde{\zeta} \circ \phi$ for any section $\phi$ of $E \rightarrow X$.

Gauge symmetries possess the following two important properties.

(i) Let $E^{\prime} \rightarrow X$ be another vector bundle and $\zeta^{\prime}$ a linear $E(X)$-valued differential operator on the $C^{\infty}(X)$-module $E^{\prime}(X)$ of sections of $E^{\prime} \rightarrow X$. Then $u_{\zeta^{\prime}(\varphi)}=\left(\zeta \circ \zeta^{\prime}\right)(\varphi)$ are also gauge symmetries of $L$ which factorize through the gauge symmetries $u_{\phi}$.

(ii) The direct and inverse second Noether theorems associate to gauge symmetries the Noether identities (henceforth NI), which variational derivatives of $L$ satisfy. ${ }^{3,4}$

Definition 1 of gauge symmetries can be generalized as follows.

Definition 2: Let a differential operator $\zeta$ in Definition 1 need not be necessarily linear. Then elements of $\operatorname{Im} \zeta$ are called generalized gauge symmetries.

However, the Noether theorems fail to hold for generalized gauge symmetries. Definition 1 of gauge symmetries has been extended to Lagrangian theory of odd fields by replacement of $C^{\infty}(X)$-modules and fiber bundles with Grassmann-graded $C^{\infty}(X)$-modules and graded manifolds whose bodies are fiber bundles, respectively. ${ }^{2,4}$

Gauge symmetries of Lagrangian field theory are thought to characterize its degeneracy. A problem is that any Lagrangian possesses gauge symmetries and, therefore, one must separate them into the trivial and non-trivial ones. Moreover, gauge symmetries can be reducible, i.e., $\operatorname{Ker} \zeta \neq 0$. Let there exist a vector bundle $E_{1} \rightarrow X$ and a linear differential operator $\zeta_{1}$ on sections of $E_{1} \rightarrow X$ taking values in $\operatorname{Ker} \zeta$. Elements $\zeta_{1}\left(\phi_{1}\right)$ of $\operatorname{Im} \zeta_{1}$ are called the first-stage gauge symmetries whose gauge parameters are sections $\phi_{1}$ of $E_{1} \rightarrow X$. Since first-stage gauge symmetries in turn can be reducible, second-stage gauge symmetries are defined, and so on. Higher-stage gauge symmetries must also be separated into the trivial and non-trivial ones. This is important because non-trivial gauge and higher-stage gauge symmetries define the BRST extension of original Lagrangian field theory for the purpose of its quantization. ${ }^{5-8}$ 
Another problem is that gauge symmetries need not form an algebra., ${ }^{5,9,10}$ The Lie bracket $\left[u_{\phi}, u_{\phi^{\prime}}\right]$ of gauge symmetries $u_{\phi}, u_{\phi^{\prime}} \in \operatorname{Im} \zeta$ is a variational symmetry, but it need not belong to $\operatorname{Im} \zeta$.

In contrast with gauge symmetries, non-trivial NI and higher-stage NI of Lagrangian field theory are well described in homology terms. ${ }^{8,11}$ Therefore, we define non-trivial gauge and higher-stage gauge symmetries as those associated to complete non-trivial NI and higher-stage NI in accordance with the inverse second Noether theorem (Definitions 9 and 11).

Lagrangian theory of even and odd fields on an $n$-dimensional smooth real manifold $X$ is adequately formulated in terms of the Grassmann-graded variational bicomplex. ${ }^{2,4,6,12}$ In accordance with general theory of NI of differential operators, ${ }^{13}$ NI of Lagrangian theory are represented by cycles of the chain complex (13), whose boundaries are treated as trivial NI and whose homology describes non-trivial NI modulo the trivial ones. ${ }^{8,11}$ Lagrangian field theory is called degenerate if its Euler-Lagrange operator satisfies non-trivial NI. The latter obey first-stage NI, and so on. To describe $(k+1)$-stage NI, let us assume that non-trivial $k$-stage NI are generated by a projective $C^{\infty}(X)$-module $\mathcal{C}_{(k)}$ of finite rank, whose elements are called complete NI. In this case, $(k+1)$-stage NI are represented by $(k+2)$-cycles of the chain complex (22) where $N=k$. If a certain homology condition (Condition 6) holds, trivial $(k+1)$-stage NI are identified with $(k+2)$-boundaries of this complex. Then its $(k+2)$-homology describes non-trivial $(k+1)$-stage NI. Degenerate Lagrangian field theory is called $k$-stage reducible if there exist non-trivial $k$-stage $\mathrm{NI}$, but all $(k+1)$-stage NI are trivial. In this case, the chain complex $(22)$ where $N=k$ is exact. It is called the Koszul-Tate (henceforth KT) complex. The nilpotentness of its boundary operator (20) is equivalent to all complete non-trivial NI and higher-stage NI. ${ }^{8,11}$

Recall that the notion of reducible NI has come from that of reducible constraints, ${ }^{6,14}$ but NI unlike constraints are differential equations. Therefore, the regularity condition for the KT complex of constraints is replaced with homology Condition 6. ${ }^{8,11}$

For the sake of simplicity, we here restrict our consideration to finitely reducible Lagrangian field theory which possesses no non-trivial $(N+1)$-stage Noether identities for some integer $N$. In this case, the KT operator (20) and the gauge operator (31) contain finite terms.

Different variants of the second Noether theorem have been suggested in order to relate reducible NI and gauge symmetries. ${ }^{3,4,6,15}$ Formulated in homology terms, the inverse second Noether theorem (Theorem 7) associates to the KT complex (22) the cochain sequence (30) 
with the ascent operator $\mathbf{u}(31){ }^{8}$ We define complete non-trivial gauge and higher-stage gauge symmetries of Lagrangian field theory as components of this ascent operator, called the gauge operator (Section IV). The gauge operator unlike the KT one is not nilpotent, unless non-trivial gauge symmetries are abelian. This is the cause why an intrinsic definition of non-trivial gauge and higher-stage gauge symmetries meets difficulties. Defined by means of the inverse second Noether theorem, non-trivial gauge and higher-stage gauge symmetries are parameterized by odd and even ghosts, but not gauge parameters. Herewith, $k$-stage ghosts form the $\left(\wedge^{n} T^{*} X\right)$-duals of the modules $\mathcal{C}_{(k+1)}$, and a $k$-stage gauge symmetry acts on $(k-1)$-stage ghosts.

For instance, the gauge operator of the gauge symmetries (3) reads

$$
\mathbf{u}=\left(d_{\lambda} c^{r}+c_{p q}^{r} a_{\lambda}^{p} c^{j}\right) \frac{\partial}{\partial a_{\lambda}^{r}},
$$

where odd ghosts $c^{r}$ are the generating elements of the exterior Grassmann algebra $\wedge \mathfrak{g}^{*}$ of the Lie coalgebra $\mathfrak{g}^{*}$. This gauge operator is not nilpotent, unless the Lie algebra $\mathfrak{g}$ is commutative. However, $\mathbf{u}(5)$ is extended to the nilpotent operator

$$
\mathbf{b}=\mathbf{u}+\gamma=\mathbf{u}-\frac{1}{2} c_{i j}^{r} c^{i} c^{j} \frac{\partial}{\partial c^{r}}
$$

by means of an additional summand $\gamma$ acting on ghosts. This nilpotent extension exists because gauge symmetries (3) form the Lie algebra (4). It is the well known BRST operator in quantum gauge theory.

Generalizing this example, we say that gauge and higher-stage gauge symmetries are algebraically closed if the gauge operator $\mathbf{u}(31)$ admits the nilpotent BRST extension $\mathbf{b}$ (41) where $k$-stage gauge symmetries are extended to $k$-stage BRST transformations acting both on $(k-1)$-stage and $k$-stage ghosts (Section $\mathrm{V}$ ). We show that this nilpotent extension exists only if the higher-stage gauge symmetry conditions hold off-shell (Proposition 12) and only if the Lie bracket of gauge symmetries is a generalized gauge symmetry factorizing through these gauge symmetries (Proposition 13). For instance, this is the case of abelian reducible Lagrangian theories and irreducible Lagrangian theories whose gauge symmetries form a Lie algebra. In abelian reducible theories, the gauge operator $\mathbf{u}$ itself is nilpotent.

In Sections VI - IX, the following characteristic examples are considered: (i) Yang-Mills supergauge theory exemplifying theory of odd fields, (ii) topological Chern-Simons theory where some gauge symmetries become trivial if $\operatorname{dim} X=3$, (iii) gauge gravitation theory whose gauge symmetries are general covariant transformations, and (iv) topological BF theory with reducible gauge symmetries. 


\section{GRASSMANN-GRADED LAGRANGIAN FIELD THEORY}

As was mentioned above, Lagrangian theory of even and odd fields is adequately formulated in terms of the variational bicomplex on fiber bundles and graded manifolds. ${ }^{2,4,12}$ Let us consider a composite bundle $F \rightarrow Y \rightarrow X$ where $F \rightarrow Y$ is a vector bundle provided with bundle coordinates $\left(x^{\lambda}, y^{i}, q^{a}\right)$. Jet manifolds $J^{r} F$ of $F \rightarrow X$ are also vector bundles $J^{r} F \rightarrow J^{r} Y$ coordinated by $\left(x^{\lambda}, y_{\Lambda}^{i}, q_{\Lambda}^{a}\right), 0 \leq|\Lambda| \leq r$, where $\Lambda=\left(\lambda_{1} \ldots \lambda_{k}\right),|\Lambda|=k$, denote symmetric multi-indices. For the sake of convenience, the value $r=0$ further stands for $F$ and $Y$. Let $\left(J^{r} Y, \mathcal{A}_{r}\right)$ be a graded manifold whose body is $J^{r} Y$ and whose structure ring $\mathcal{A}_{r}$ of graded functions consists of sections of the exterior bundle

$$
\wedge\left(J^{r} F\right)^{*}=\mathbb{R} \oplus\left(J^{r} F\right)^{*} \oplus \stackrel{2}{\wedge}\left(J^{r} F\right)^{*} \oplus \cdots,
$$

where $\left(J^{r} F\right)^{*}$ is the dual of $J^{r} F \rightarrow J^{r} Y$. The local odd basis for this ring is $\left\{c_{\Lambda}^{a}\right\}, 0 \leq|\Lambda| \leq$ $r$. Let $\mathcal{S}_{r}^{*}[F ; Y]$ be the differential graded algebra (henceforth DGA) of graded differential forms on the graded manifold $\left(J^{r} Y, \mathcal{A}_{r}\right)$. The inverse system of jet manifolds $J^{r-1} Y \leftarrow J^{r} Y$ yields the direct system of DGAs

$$
\mathcal{S}^{*}[F ; Y] \longrightarrow \mathcal{S}_{1}^{*}[F ; Y] \longrightarrow \cdots \mathcal{S}_{r}^{*}[F ; Y] \longrightarrow \cdots
$$

Its direct limit $\mathcal{S}_{\infty}^{*}[F ; Y]$ is the DGA of all graded differential forms on graded manifolds $\left(J^{r} Y, \mathcal{A}_{r}\right)$. Recall the formulas

$$
\phi \wedge \phi^{\prime}=(-1)^{|\phi|\left|\phi^{\prime}\right|+[\phi]\left[\phi^{\prime}\right]} \phi^{\prime} \wedge \phi, \quad d\left(\phi \wedge \phi^{\prime}\right)=d \phi \wedge \phi^{\prime}+(-1)^{|\phi|} \phi \wedge d \phi,
$$

where $[\phi]$ denotes the Grassmann parity. The DGA $S_{\infty}^{*}[F ; Y]$ contains the subalgebra $\mathcal{O}_{\infty}^{*} Y$ of all exterior forms on jet manifolds $J^{r} Y$. It is an $\mathcal{O}_{\infty}^{0} Y$-algebra locally generated by elements $\left(c_{\Lambda}^{a}, d x^{\lambda}, d y_{\Lambda}^{i}, d c_{\Lambda}^{a}\right), 0 \leq|\Lambda|$. The collective symbol $\left(s^{A}\right)$ further stands for the tuple $\left(y^{i}, c^{a}\right)$, called the local basis for the DGA $\mathcal{S}_{\infty}^{*}[F ; Y]$. We denote $[A]=\left[s^{A}\right]=\left[s_{\Lambda}^{A}\right]$.

The DGA $\mathcal{S}_{\infty}^{*}[F ; Y]$ is decomposed into the Grassmann-graded variational bicomplex of modules $\mathcal{S}_{\infty}^{k, r}[F ; Y]$ of $r$-horizontal and $k$-contact graded forms locally generated by oneforms $d x^{\lambda}$ and $\theta_{\Lambda}^{A}=d s_{\Lambda}^{A}-s_{\lambda+\Lambda}^{A} d x^{\lambda}$. It contains the variational subcomplex

$$
0 \rightarrow \mathbb{R} \longrightarrow \mathcal{S}_{\infty}^{0}[F ; Y] \stackrel{d_{H}}{\longrightarrow} \mathcal{S}_{\infty}^{0,1}[F ; Y] \cdots \stackrel{d_{H}}{\longrightarrow} \mathcal{S}_{\infty}^{0, n}[F ; Y] \stackrel{\delta}{\longrightarrow} \mathcal{S}_{\infty}^{1, n}[F ; Y],
$$

where

$$
d_{H}(\phi)=d x^{\lambda} \wedge d_{\lambda} \phi, \quad d_{\lambda}=\partial_{\lambda}+\sum_{0 \leq|\Lambda|} s_{\lambda \Lambda}^{A} \partial_{A}^{\Lambda}
$$


is the total differential and

$$
\delta L=\theta^{A} \wedge \mathcal{E}_{A} d^{n} x=\sum_{0 \leq|\Lambda|}(-1)^{|\Lambda|} \theta^{A} \wedge d_{\Lambda}\left(\partial_{A}^{\Lambda} \mathcal{L}\right) d^{n} x, \quad d_{\Lambda}=d_{\lambda_{1}} \cdots d_{\lambda_{k}},
$$

is the variational operator. Lagrangians and Euler-Lagrange operators are defined as even elements

$$
L=\mathcal{L} d^{n} x \in \mathcal{S}_{\infty}^{0, n}[F ; Y], \quad \delta L=\theta^{A} \wedge \mathcal{E}_{A} d^{n} x \in \mathcal{S}_{\infty}^{1, n}[F ; Y]
$$

Further, we call a pair $\left(\mathcal{S}_{\infty}^{*}[F ; Y], L\right)$ the Lagrangian field theory.

Cohomology of the variational bicomplex has been obtained. ${ }^{2,12}$ Let us mention the following results.

Proposition 3: Any variationally trivial (i.e., $\delta$-closed) graded density $L \in \mathcal{S}_{\infty}^{0, n}[F ; Y]$ takes the form $L=d_{H} \psi+h_{0} \varphi$, where $\varphi$ is a closed exterior $n$-form on $Y$ where $h_{0}\left(d y^{i}\right)=$ $y_{\mu}^{i} d x^{\mu}$. In particular, any odd variationally trivial graded density is $d_{H^{-}}$-exact.

Proposition 4: The form $d L-\delta L$ is $d_{H}$-exact for any graded density $L \in \mathcal{S}_{\infty}^{0, n}[F ; Y]$.

In order to treat symmetries of Lagrangian field theory $\left(\mathcal{S}_{\infty}^{*}[F ; Y], L\right)$ in a very general setting, we consider graded derivations of the $\mathbb{R}$-ring $\mathcal{S}_{\infty}^{0}[F ; Y]{ }^{2}$ They take the form

$$
\left.\vartheta=\vartheta^{\lambda} \partial_{\lambda}+\sum_{0 \leq|\Lambda|} \vartheta_{\Lambda}^{A} \partial_{A}^{\Lambda}, \quad \partial_{A}^{\Lambda}\left(s_{\Sigma}^{B}\right)=\partial_{A}^{\Lambda}\right\rfloor d s_{\Sigma}^{B}=\delta_{A}^{B} \delta_{\Sigma}^{\Lambda}
$$

Any graded derivation $\vartheta(7)$ yields the Lie derivative

$$
\left.\left.\mathbf{L}_{\vartheta} \phi=\vartheta\right\rfloor d \phi+d(\vartheta\rfloor \phi\right)
$$

of the DGA $\mathcal{S}_{\infty}^{*}[F ; Y]$ which obeys the relations

$$
\left.\left.\mathbf{L}_{\vartheta} \phi=\vartheta\right\rfloor d \phi+d(\vartheta\rfloor \phi\right), \quad \mathbf{L}_{\vartheta}\left(\phi \wedge \phi^{\prime}\right)=\mathbf{L}_{\vartheta}(\phi) \wedge \phi^{\prime}+(-1)^{[\vartheta][\phi]} \phi \wedge \mathbf{L}_{\vartheta}\left(\phi^{\prime}\right)
$$

A graded derivation $\vartheta(7)$ is called contact if the Lie derivative $\mathbf{L}_{\vartheta}$ preserves the ideal of contact graded forms of the DGA $\mathcal{S}_{\infty}^{*}[F ; Y]$. Any contact graded derivation admits the decomposition

$$
\vartheta=\vartheta_{H}+\vartheta_{V}=\vartheta^{\lambda} d_{\lambda}+\left(\vartheta^{A} \partial_{A}+\sum_{0<|\Lambda|} d_{\Lambda} \vartheta^{A} \partial_{A}^{\Lambda}\right)
$$

into the horizontal and vertical parts $\vartheta_{H}$ and $\vartheta_{V}$.

Given a graded density $L(6)$, a contact graded derivation $\vartheta(8)$ is said to be its variational symmetry if the Lie derivative $\mathbf{L}_{\vartheta} L$ of $L$ is a variationally trivial graded density. If $\mathbf{L}_{\vartheta} L=0$, a variational symmetry of $L$ is called its exact symmetry. 
Proposition 5: A contact graded derivation $\vartheta(8)$ is a variational symmetry iff its vertical part $\vartheta_{V}$ is also. ${ }^{2}$

Therefore, we further restrict our consideration to vertical contact graded derivations $\vartheta$. Such a derivation is the jet prolongation

$$
\vartheta=v^{A} \partial_{A}+\sum_{0<|\Lambda|} d_{\Lambda} v^{A} \partial_{A}^{\Lambda}
$$

of its restriction $v=v^{A} \partial_{A}$ to the $\operatorname{ring} \mathcal{S}_{\infty}^{0}[F ; Y]$. Therefore, the relations

$$
\begin{aligned}
& \left.\vartheta\rfloor d_{H} \phi=-d_{H}(\vartheta\rfloor \phi\right), \quad \phi \in \mathcal{S}_{\infty}^{*}[F ; Y] \\
& \mathbf{L}_{\vartheta}\left(d_{H} \phi\right)=d_{H}\left(\mathbf{L}_{\vartheta} \phi\right)
\end{aligned}
$$

hold. By virtue of the relation (10) and Proposition 4, the Lie derivative $\mathbf{L}_{\vartheta} L$ of any graded density $L$ admits the decomposition

$$
\left.\left.\left.\left.\mathbf{L}_{\vartheta} L=\vartheta\right\rfloor d L=\vartheta\right\rfloor \delta L+\vartheta\right\rfloor(d L-\delta L)=v\right\rfloor \delta L+d_{H} \sigma=v^{A} \mathcal{E}_{A} d^{n} x+d_{H} \sigma,
$$

called the first variational formula. A glance at the expression (12) shows that $\vartheta(9)$ is a variational symmetry of $L$ iff the graded density $v\rfloor \delta L$ is variationally trivial.

By virtue of the relation (11), any graded derivation (9) is a variational symmetry of a variationally trivial graded density. It follows that variational symmetries of a graded density constitute a real Lie algebra $\mathcal{G}_{L}$.

A graded derivation $\vartheta(9)$ is called nilpotent if $\mathbf{L}_{\vartheta}\left(\mathbf{L}_{\vartheta} \phi\right)=0$ for any horizontal form $\phi \in \mathcal{S}_{\infty}^{0 * *}[F ; Y]$. One can show that $\vartheta(9)$ is nilpotent only if it is odd and iff $\vartheta(v)=0 .{ }^{2}$

For the sake of simplicity, the common symbol $v$ further stands for the graded derivation $\vartheta(9)$, its first term $v$, and the Lie derivative $\mathbf{L}_{\vartheta}$. We agree to call $v$ the graded derivation of the DGA $\mathcal{S}_{\infty}^{*}[F ; Y]$. Its right graded derivations $\overleftarrow{v}=\overleftarrow{\partial}_{A} v^{A}$ are also considered

\section{NOETHER IDENTITIES}

To describe reducible NI of Lagrangian field theory $\left(\mathcal{S}_{\infty}^{*}[F ; Y], L\right),{ }^{8,11}$ let us introduce the following notation. Given a vector bundle $E \rightarrow X$, we call

$$
\bar{E}=E^{*} \otimes \wedge^{n} T^{*} X
$$

the density-dual of $E$. The density-dual of a graded vector bundle $E=E^{0} \oplus E^{1}$ is $\bar{E}=$ $\bar{E}^{1} \oplus \bar{E}^{0}$. Given a graded vector bundle $E=E^{0} \oplus E^{1}$ over $Y$, we consider the composite 
bundle $E \rightarrow E^{0} \rightarrow X$ and denote $\mathcal{P}_{\infty}^{*}[E ; Y]=\mathcal{S}_{\infty}^{*}\left[E ; E^{0}\right]$. Let $V F$ be the vertical tangent bundle of $F \rightarrow X$, the density-dual of the vector bundle $V F \rightarrow F$ is

$$
\overline{V F}=V^{*} F \underset{F}{\otimes} \wedge{ }^{n} T^{*} X
$$

Let us enlarge $\mathcal{S}_{\infty}^{*}[F ; Y]$ to the DGA $\mathcal{P}_{\infty}^{*}[\overline{V F} ; Y]$ with the local basis $\left(s^{A}, \bar{s}_{A}\right),\left[\bar{s}_{A}\right]=$ $([A]+1) \bmod 2$. Its elements $\bar{s}_{A}$ are called antifields of antifield number $\operatorname{Ant}\left[\bar{s}_{A}\right]=1$. The DGA $\mathcal{P}_{\infty}^{*}[\overline{V F} ; Y]$ is endowed with the nilpotent right graded derivation $\bar{\delta}=\overleftarrow{\partial}{ }^{A} \mathcal{E}_{A}$, where $\mathcal{E}_{A}$ are the variational derivatives (6). Then we have the chain complex

$$
0 \leftarrow \operatorname{Im} \bar{\delta} \stackrel{\bar{\delta}}{\longleftarrow} \mathcal{P}_{\infty}^{0, n}[\overline{V F} ; Y]_{1} \stackrel{\bar{\delta}}{\longleftarrow} \mathcal{P}_{\infty}^{0, n}[\overline{V F} ; Y]_{2}
$$

of graded densities of antifield number $\leq 2$. Its one-cycles

$$
\bar{\delta} \Phi=0, \quad \Phi=\sum_{0 \leq|\Lambda|} \Phi^{A, \Lambda} \bar{s}_{\Lambda A} d^{n} x \in \mathcal{P}_{\infty}^{0, n}[\overline{V F} ; Y]_{1},
$$

define NI of Lagrangian field theory $\left(\mathcal{S}_{\infty}^{*}[F ; Y], L\right)$. In particular, one-chains $\Phi \in \mathcal{P}_{\infty}^{0, n}[\overline{V F} ; Y]_{1}$ are necessarily NI if they are boundaries. Therefore, these NI are called trivial. Accordingly, non-trivial NI modulo the trivial ones are associated to elements of the first homology $H_{1}(\bar{\delta})$ of the complex (13).

Non-trivial NI obey first-stage NI. To describe them, let us assume that the module $H_{1}(\bar{\delta})$ is finitely generated. Namely, there exists a projective $C^{\infty}(X)$-module $\mathcal{C}_{(0)} \subset H_{1}(\bar{\delta})$ of finite rank possessing the local basis $\left\{\Delta_{r}\right\}$ such that any element $\Phi \in H_{1}(\bar{\delta})$ factorizes as

$$
\Phi=\sum_{0 \leq|\Xi|} G^{r, \Xi} d_{\Xi} \Delta_{r} d^{n} x, \quad \Delta_{r}=\sum_{0 \leq|\Lambda|} \Delta_{r}^{A, \Lambda} \bar{s}_{\Lambda A}, \quad G^{r, \Xi}, \Delta_{r}^{A, \Lambda} \in \mathcal{S}_{\infty}^{0}[F ; Y],
$$

through elements of $\mathcal{C}_{(0)}$. Thus, all non-trivial NI (14) result from the NI

$$
\bar{\delta} \Delta_{r}=\sum_{0 \leq|\Lambda|} \Delta_{r}^{A, \Lambda} d_{\Lambda} \mathcal{E}_{A}=0,
$$

called the complete NI. By virtue of the Serre-Swan theorem, ${ }^{11}$ the module $\mathcal{C}_{(0)}$ is isomorphic to the $C^{\infty}(X)$-module of sections of the density-dual $\bar{E}_{0}$ of some graded vector bundle $E_{0} \rightarrow X$. Let us enlarge $\mathcal{P}_{\infty}^{*}[\overline{V F} ; Y]$ to the DGA

$$
\overline{\mathcal{P}}_{\infty}^{*}\{0\}=\mathcal{P}_{\infty}^{*}\left[\overline{V F} \oplus_{Y} \bar{E}_{0} ; Y\right]
$$


possessing the local basis $\left(s^{A}, \bar{s}_{A}, \bar{c}_{r}\right)$ of Grassmann parity $\left[\bar{c}_{r}\right]=\left(\left[\Delta_{r}\right]+1\right) \bmod 2$ and of antifield number Ant $\left[\bar{c}_{r}\right]=2$. This DGA is provided with the odd right graded derivation $\delta_{0}=\bar{\delta}+\overleftarrow{\partial}^{r} \Delta_{r}$ which is nilpotent iff the NI (16) hold. Then we have the chain complex

$$
0 \leftarrow \operatorname{Im} \bar{\delta} \stackrel{\bar{\delta}}{\leftarrow} \mathcal{P}_{\infty}^{0, n}[\overline{V F} ; Y]_{1} \stackrel{\delta_{0}}{\leftarrow} \overline{\mathcal{P}}_{\infty}^{0, n}\{0\}_{2} \stackrel{\delta_{0}}{\leftarrow} \overline{\mathcal{P}}_{\infty}^{0, n}\{0\}_{3}
$$

of graded densities of antifield number $\leq 3$. It possesses trivial homology $H_{0}\left(\delta_{0}\right)$ and $H_{1}\left(\delta_{0}\right)$. Its two-cycles define the first-stage NI

$$
\begin{aligned}
& \delta_{0} \Phi=0, \quad \Phi=G+H=\sum_{0 \leq|\Lambda|} G^{r, \Lambda} \bar{c}_{\Lambda r} d^{n} x+\sum_{0 \leq|\Lambda|,|\Sigma|} H^{(A, \Lambda)(B, \Sigma)} \bar{s}_{\Lambda A} \bar{s}_{\Sigma B} d^{n} x, \\
& \sum_{0 \leq|\Lambda|} G^{r, \Lambda} d_{\Lambda} \Delta_{r} d^{n} x=-\bar{\delta} H .
\end{aligned}
$$

However, the converse need not be true. One can show that NI (18) are cycles iff any $\bar{\delta}$ cycle $\Phi \in \mathcal{P}_{\infty}^{0, n}[\overline{V F} ; Y]_{2}$ is a $\delta_{0}$-boundary. ${ }^{11}$ Any boundary $\Phi \in \overline{\mathcal{P}}_{\infty}^{0, n}\{0\}_{2}$ necessarily defines first-stage NI (18), called trivial. Accordingly, non-trivial first-stage NI modulo the trivial ones are identified with elements of the second homology $H_{2}\left(\delta_{0}\right)$ of the complex $(17)$.

Non-trivial first-stage NI obey second-stage NI, and so on. Iterating the arguments, one can characterize $N$-stage reducible Lagrangian field theory $\left(\mathcal{S}_{\infty}^{*}[F ; Y], L\right)$ as follows. There are graded vector bundles $E_{0}, \ldots, E_{N}$ over $X$, and the $\mathrm{DGA}_{\infty} \mathcal{P}_{\infty}^{*}[\overline{V F} ; Y]$ is enlarged to the DGA

$$
\overline{\mathcal{P}}_{\infty}^{*}\{N\}=\mathcal{P}_{\infty}^{*}\left[\overline{V F} \underset{Y}{\oplus} \bar{E}_{0} \underset{Y}{\oplus} \cdots \oplus_{Y}^{\oplus} \bar{E}_{N} ; Y\right]
$$

with the local basis $\left(s^{A}, \bar{s}_{A}, \bar{c}_{r}, \bar{c}_{r_{1}}, \ldots, \bar{c}_{r_{N}}\right)$ of antifield number Ant $\left[\bar{c}_{r_{k}}\right]=k+2$. The DGA (19) is provided with the nilpotent right graded derivation

$$
\begin{aligned}
\delta_{K T} & =\overleftarrow{\partial}{ }^{A} \mathcal{E}_{A}+\sum_{0 \leq|\Lambda|} \overleftarrow{\partial}^{r} \Delta_{r}^{A, \Lambda} \bar{s}_{\Lambda A}+\sum_{1 \leq k \leq N} \overleftarrow{\partial}^{r_{k}} \Delta_{r_{k}}, \\
\Delta_{r_{k}} & =\sum_{0 \leq|\Lambda|} \Delta_{r_{k}}^{r_{k-1}, \Lambda} \bar{c}_{\Lambda r_{k-1}}+\sum_{0 \leq|\Sigma|,|\Xi|}\left(h_{r_{k}}^{\left(r_{k-2}, \Sigma\right)(A, \Xi)} \bar{c}_{\Sigma r_{k-2}} \bar{s}_{\Xi A}+\ldots\right)
\end{aligned}
$$

of antifield number -1 , where the index $k=-1$ stands for $\bar{s}_{A}$. It is called the KT operator. With this graded derivation, the module $\overline{\mathcal{P}}_{\infty}^{0, n}\{N\}_{\leq N+3}$ of densities of antifield number $\leq(N+3)$ is decomposed into the exact $\mathrm{KT}$ chain complex

$$
\begin{aligned}
0 \leftarrow & \operatorname{Im} \bar{\delta} \stackrel{\bar{\delta}}{\longleftarrow} \mathcal{P}_{\infty}^{0, n}[\overline{V F} ; Y]_{1} \stackrel{\delta_{0}}{\longleftarrow} \overline{\mathcal{P}}_{\infty}^{0, n}\{0\}_{2} \stackrel{\delta_{1}}{\longleftarrow} \overline{\mathcal{P}}_{\infty}^{0, n}\{1\}_{3} \ldots \\
& \stackrel{\delta_{N-1}}{\longleftarrow} \overline{\mathcal{P}}_{\infty}^{0, n}\{N-1\}_{N+1} \stackrel{\delta_{K T}}{\longleftarrow} \overline{\mathcal{P}}_{\infty}^{0, n}\{N\}_{N+2} \stackrel{\delta_{K T}}{\longleftarrow} \overline{\mathcal{P}}_{\infty}^{0, n}\{N\}_{N+3}
\end{aligned}
$$


which satisfies the following homology condition.

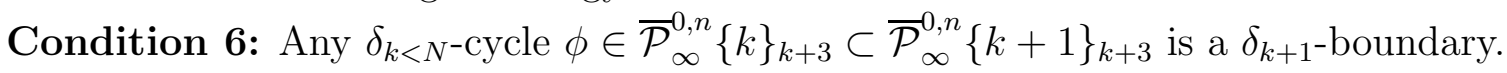

Given the KT complex (22), the nilpotentness $\delta_{K T}^{2}=0$ of its boundary operator (20) is equivalent to the complete non-trivial NI (16) and the complete non-trivial $(1 \leq k \leq N)$ stage NI

$$
\sum_{0 \leq|\Lambda|} \Delta_{r_{k}}^{r_{k-1}, \Lambda} d_{\Lambda}\left(\sum_{0 \leq|\Sigma|} \Delta_{r_{k-1}}^{r_{k-2}, \Sigma} \bar{c}_{\Sigma r_{k-2}}\right)=-\bar{\delta}\left(\sum_{0 \leq|\Sigma|,|\Xi|} h_{r_{k}}^{\left(r_{k-2}, \Sigma\right)(A, \Xi)} \bar{c}_{\Sigma r_{k-2}} \bar{s}_{\Xi A}\right) .
$$

\section{GAUGE SYMMETRIES}

We define non-trivial gauge and higher-stage gauge symmetries of Lagrangian field theory $\left(\mathcal{S}_{\infty}^{*}[F ; Y], L\right)$ as those associated to the NI (16) and higher-stage NI (23) by means of the inverse second Noether theorem.

Let us start with the following notation. Given the DGA $\overline{\mathcal{P}}_{\infty}^{*}\{N\}$ (19), we consider the DGA

$$
\mathcal{P}_{\infty}^{*}\{N\}=\mathcal{P}_{\infty}^{*}\left[F \underset{Y}{\oplus} E_{0} \underset{Y}{\oplus} \cdots \oplus_{Y} E_{N} ; Y\right],
$$

possessing the local basis $\left(s^{A}, c^{r}, c^{r_{1}}, \ldots, c^{r_{N}}\right),\left[c^{r_{k}}\right]=\left(\left[\bar{c}_{r_{k}}\right]+1\right) \bmod 2$, and the DGA

$$
P_{\infty}^{*}\{N\}=\mathcal{P}_{\infty}^{*}\left[\overline{V F} \oplus_{Y} E_{0} \oplus \cdots \oplus_{Y} E_{N} \underset{Y}{\oplus} \bar{E}_{0} \underset{Y}{\oplus} \cdots \underset{Y}{\oplus} \bar{E}_{N} ; Y\right]
$$

with the local basis $\left(s^{A}, \bar{s}^{A}, c^{r}, c^{r_{1}}, \ldots, c^{r_{N}}, \bar{c}_{r}, \bar{c}_{r_{1}}, \ldots, \bar{c}_{r_{N}}\right)$. Their elements $c^{r_{k}}$ are called $k$ stage ghosts of ghost number $\operatorname{gh}\left[c^{r_{k}}\right]=k+1$ and antifield number $\operatorname{Ant}\left[c^{r_{k}}\right]=-(k+1)$. The $C^{\infty}(X)$-module $\mathcal{C}^{(k)}$ of $k$-stage ghosts is the density dual of the module $\left.\mathcal{C}_{(k+1}\right)$ of $(k+1)$-stage antifields. The DGAs $\overline{\mathcal{P}}_{\infty}^{*}\{N\}$ (19) and $\mathcal{P}_{\infty}^{*}\{N\}$ (24) are subalgebras of $P_{\infty}^{*}\{N\}$ (25). The KT operator $\delta_{K T}(20)$ is naturally extended to a graded derivation of the DGA $P_{\infty}^{*}\{N\}$.

We refer to the following formulas in the sequel. ${ }^{3}$ Any graded form $\phi \in \mathcal{S}_{\infty}^{*}[F ; Y]$ and any finite tuple $\left(f^{\Lambda}\right), 0 \leq|\Lambda|$, of local graded functions $f^{\Lambda} \in \mathcal{S}_{\infty}^{0}[F ; Y]$ obey the relations

$$
\begin{aligned}
& \sum_{0 \leq|\Lambda| \leq k} f^{\Lambda} d_{\Lambda} \phi \wedge d^{n} x=\sum_{0 \leq|\Lambda|}(-1)^{|\Lambda|} d_{\Lambda}\left(f^{\Lambda}\right) \phi \wedge d^{n} x+d_{H} \sigma, \\
& \sum_{0 \leq|\Lambda| \leq k}(-1)^{|\Lambda|} d_{\Lambda}\left(f^{\Lambda} \phi\right)=\sum_{0 \leq|\Lambda| \leq k} \eta(f)^{\Lambda} d_{\Lambda} \phi, \\
& \eta(f)^{\Lambda}=\sum_{0 \leq|\Sigma| \leq k-|\Lambda|}(-1)^{|\Sigma+\Lambda|} \frac{(|\Sigma+\Lambda|) !}{|\Sigma| !|\Lambda| !} d_{\Sigma} f^{\Sigma+\Lambda}, \\
& \eta(\eta(f))^{\Lambda}=f^{\Lambda} .
\end{aligned}
$$


Theorem 7: Given the KT complex (22), the module of graded densities $\mathcal{P}_{\infty}^{0, n}\{N\}$ is decomposed into the cochain sequence

$$
\begin{aligned}
& 0 \rightarrow \mathcal{S}_{\infty}^{0, n}[F ; Y] \stackrel{\mathbf{u}}{\longrightarrow} \mathcal{P}_{\infty}^{0, n}\{N\}^{1} \stackrel{\mathbf{u}}{\longrightarrow} \mathcal{P}_{\infty}^{0, n}\{N\}^{2} \stackrel{\mathbf{u}}{\longrightarrow} \cdots \\
& \mathbf{u}=u+u^{(1)}+\cdots+u^{(N)}=u^{A} \frac{\partial}{\partial s^{A}}+u^{r} \frac{\partial}{\partial c^{r}}+\cdots+u^{r_{N-1}} \frac{\partial}{\partial c^{r_{N-1}}}
\end{aligned}
$$

graded in ghost number. Its ascent operator $\mathbf{u}(31)$ is an odd graded derivation of ghost number 1 where $u(36)$ is a variational symmetry of a Lagrangian $L$ and the graded derivations $u_{(k)}(38), k=1, \ldots, N$, obey the relations $(37)$.

Proof: Given the KT operator (20), let us extend an original Lagrangian $L$ to the Lagrangian

$$
L_{e}=L+L_{1}=L+\sum_{0 \leq k \leq N} c^{r_{k}} \Delta_{r_{k}} d^{n} x=L+\delta_{K T}\left(\sum_{0 \leq k \leq N} c^{r_{k}} \bar{c}_{r_{k}} d^{n} x\right)
$$

of zero antifield number. It is readily observed that the $\mathrm{KT}$ operator $\delta_{K T}$ is a variational symmetry of $L_{e}$. Since $\delta_{K T}$ is odd, it follows from the first variational formula (12) and Proposition 3 that

$$
\begin{aligned}
& {\left[\frac{\overleftarrow{\delta} \mathcal{L}_{e}}{\delta \bar{s}_{A}} \mathcal{E}_{A}+\sum_{0 \leq k \leq N} \frac{\overleftarrow{\delta} \mathcal{L}_{e}}{\delta \bar{c}_{r_{k}}} \Delta_{r_{k}}\right] d^{n} x=\left[v^{A} \mathcal{E}_{A}+\sum_{0 \leq k \leq N} v^{r_{k}} \frac{\delta \mathcal{L}_{e}}{\delta c^{r_{k}}}\right] d^{n} x=d_{H} \sigma} \\
& v^{A}=\frac{\overleftarrow{\delta} \mathcal{L}_{e}}{\delta \bar{s}_{A}}=u^{A}+w^{A}=\sum_{0 \leq|\Lambda|} c_{\Lambda}^{r} \eta\left(\Delta_{r}^{A}\right)^{\Lambda}+\sum_{1 \leq i \leq N} \sum_{0 \leq|\Lambda|} c_{\Lambda}^{r_{i}} \eta\left(\overleftarrow{\partial}{ }^{A}\left(h_{r_{i}}\right)\right)^{\Lambda} \\
& v^{r_{k}}=\frac{\overleftarrow{\delta} \mathcal{L}_{e}}{\delta \bar{c}_{r_{k}}}=u^{r_{k}}+w^{r_{k}}=\sum_{0 \leq|\Lambda|} c_{\Lambda}^{r_{k+1}} \eta\left(\Delta_{r_{k+1}}^{r_{k}}\right)^{\Lambda}+\sum_{k+1<i \leq N} \sum_{0 \leq|\Lambda|} c_{\Lambda}^{r_{i}} \eta\left(\overleftarrow{\partial}^{r_{k}}\left(h_{r_{i}}\right)\right)^{\Lambda}
\end{aligned}
$$

The equality (33) falls into the set of equalities

$$
\begin{aligned}
& \frac{\overleftarrow{\delta}\left(c^{r} \Delta_{r}\right)}{\delta \bar{s}_{A}} \mathcal{E}_{A} d^{n} x=u^{A} \mathcal{E}_{A} d^{n} x=d_{H} \sigma_{0} \\
& {\left[\frac{\overleftarrow{\delta}\left(c^{r_{k}} \Delta_{r_{k}}\right)}{\delta \bar{s}_{A}} \mathcal{E}_{A}+\sum_{0 \leq i<k} \frac{\overleftarrow{\delta}\left(c^{r_{k}} \Delta_{r_{k}}\right)}{\delta \bar{c}_{r_{i}}} \Delta_{r_{i}}\right] d^{n} x=d_{H} \sigma_{k}, \quad k=1, \ldots, N}
\end{aligned}
$$

A glance at the equality (34) shows that, by virtue of the first variational formula (12), the odd graded derivation

$$
u=u^{A} \frac{\partial}{\partial s^{A}}, \quad u^{A}=\sum_{0 \leq|\Lambda|} c_{\Lambda}^{r} \eta\left(\Delta_{r}^{A}\right)^{\Lambda}
$$


of $\mathcal{P}^{0}\{0\}$ is a variational symmetry of a Lagrangian L. Every equality (35) falls into a set of equalities graded by the polynomial degree in antifields. Let us consider that of them linear in antifields $\bar{c}_{r_{k-2}}$. We have

$$
\begin{aligned}
& {\left[\frac{\overleftarrow{\delta}}{\delta \bar{s}_{A}}\left(c^{r_{k}} \sum_{0 \leq|\Sigma|,|\Xi|} h_{r_{k}}^{\left(r_{k-2}, \Sigma\right)(A, \Xi)} \bar{c}_{\Sigma r_{k-2}} \bar{s}_{\Xi A}\right) \mathcal{E}_{A}+\right.} \\
& \left.\quad \frac{\overleftarrow{\delta}}{\delta \bar{c}_{r_{k-1}}}\left(c^{r_{k}} \sum_{0 \leq|\Sigma|} \Delta_{r_{k}}^{r_{k-1}^{\prime}, \Sigma} \bar{c}_{\Sigma r_{k-1}^{\prime}}\right) \sum_{0 \leq|\Xi|} \Delta_{r_{k-1}}^{r_{k-2}, \Xi_{\bar{c}}} \bar{\Xi}_{\Xi r_{k-2}}\right] d^{n} x=d_{H} \sigma_{k}
\end{aligned}
$$

This equality is brought into the form

$$
\left[\sum_{0 \leq|\Xi|}(-1)^{|\Xi|} d_{\Xi}\left(c^{r_{k}} \sum_{0 \leq|\Sigma|} h_{r_{k}}^{\left(r_{k-2}, \Sigma\right)(A, \Xi)} \bar{c}_{\Sigma r_{k-2}}\right) \mathcal{E}_{A}+u^{r_{k-1}} \sum_{0 \leq|\Xi|} \Delta_{r_{k-1}}^{r_{k-2}, \Xi} \bar{c}_{\Xi r_{k-2}}\right] d^{n} x=d_{H} \sigma_{k} .
$$

Using the relation (26), we obtain the equality

$$
\left[\sum_{0 \leq|\Xi|} c^{r_{k}} \sum_{0 \leq|\Sigma|} h_{r_{k}}^{\left(r_{k-2}, \Sigma\right)(A, \Xi)} \bar{c}_{\Sigma r_{k-2}} d_{\Xi} \mathcal{E}_{A}+u^{r_{k-1}} \sum_{0 \leq|\Xi|} \Delta_{r_{k-1}}^{r_{k-2}, \Xi} \bar{c}_{\Xi r_{k-2}}\right] d^{n} x=d_{H} \sigma_{k}^{\prime}
$$

The variational derivative of both its sides with respect to $\bar{c}_{r_{k-2}}$ leads to the relation

$$
\begin{aligned}
& \sum_{0 \leq|\Sigma|} d_{\Sigma} u^{r_{k-1}} \frac{\partial}{\partial c_{\Sigma}^{r_{k-1}}} u^{r_{k-2}}=\bar{\delta}\left(\alpha^{r_{k-2}}\right), \\
& \alpha^{r_{k-2}}=-\sum_{0 \leq|\Sigma|} \eta\left(h_{r_{k}}^{\left(r_{k-2}\right)(A, \Xi)}\right)^{\Sigma} d_{\Sigma}\left(c^{r_{k}} \bar{s}_{\Xi A}\right),
\end{aligned}
$$

which the odd graded derivation

$$
u^{(k)}=u^{r_{k-1}} \frac{\partial}{\partial c^{r_{k-1}}}, \quad u^{r_{k-1}}=\sum_{0 \leq|\Lambda|} c_{\Lambda}^{r_{k}} \eta\left(\Delta_{r_{k}}^{r_{k-1}}\right)^{\Lambda}, \quad k=1, \ldots, N
$$

satisfies. Graded derivations $u(36)$ and $u^{(k)}(38)$ are assembled into the ascent operator $\mathbf{u}$ (31) of the cochain sequence (30).

A glance at the expression (36) shows that the variational symmetry $u$ is a linear differential operator on the $C^{\infty}(X)$-module $\mathcal{C}^{(0)}$ of ghosts with values in the real space $\mathcal{G}_{L}$ of variational symmetries. Following Definition 1 extended to Lagrangian theories of odd fields, we call $u$ (36) the gauge symmetry of a Lagrangian $L$ which is associated to the NI (16). This association is unique due to the following.

Proposition 8: The variational derivative of the equality (34) with respect to ghosts $c^{r}$ leads to the equality

$$
\delta_{r}\left(u^{A} \mathcal{E}_{A} d^{n} x\right)=\sum_{0 \leq|\Lambda|}(-1)^{|\Lambda|} d_{\Lambda}\left(\eta\left(\Delta_{r}^{A}\right)^{\Lambda} \mathcal{E}_{A}\right)=\sum_{0 \leq|\Lambda|}(-1)^{|\Lambda|} \eta\left(\eta\left(\Delta_{r}^{A}\right)\right)^{\Lambda} d_{\Lambda} \mathcal{E}_{A}=0
$$


which reproduces the complete non-trivial NI (16) by means of the relation (29).

Moreover, the gauge symmetry $u(36)$ is complete in the following sense. Let

$$
\sum_{0 \leq|\Xi|} C^{R} G_{R}^{r, \Xi} d_{\Xi} \Delta_{r} d^{n} x
$$

be some projective $C^{\infty}(X)$-module of finite rank of non-trivial NI (15) parameterized by the corresponding ghosts $C^{R}$. We have the equalities

$$
\begin{aligned}
0= & \sum_{0 \leq|\Xi|} C^{R} G_{R}^{r, \Xi} d_{\Xi}\left(\sum_{0 \leq|\Lambda|} \Delta_{r}^{A, \Lambda} d_{\Lambda} \mathcal{E}_{A}\right) d^{n} x= \\
& \sum_{0 \leq|\Lambda|}\left(\sum_{0 \leq|\Xi|} \eta\left(G_{R}^{r}\right)^{\Xi} C_{\Xi}^{R}\right) \Delta_{r}^{A, \Lambda} d_{\Lambda} \mathcal{E}_{A} d^{n} x+d_{H}(\sigma)= \\
& \sum_{0 \leq|\Lambda|}(-1)^{|\Lambda|} d_{\Lambda}\left(\Delta_{r}^{A, \Lambda} \sum_{0 \leq|\Xi|} \eta\left(G_{R}^{r}\right)^{\Xi} C_{\Xi}^{R}\right) \mathcal{E}_{A} d^{n} x+d_{H} \sigma= \\
& \sum_{0 \leq|\Lambda|} \eta\left(\Delta_{r}^{A}\right)^{\Lambda} d_{\Lambda}\left(\sum_{0 \leq|\Xi|} \eta\left(G_{R}^{r}\right)^{\Xi} C_{\Xi}^{R}\right) \mathcal{E}_{A} d^{n} x+d_{H} \sigma= \\
& \sum_{0 \leq|\Lambda|} u_{r}^{A, \Lambda} d_{\Lambda}\left(\sum_{0 \leq|\Xi|} \eta\left(G_{R}^{r}\right)^{\Xi} C_{\Xi}^{R}\right) \mathcal{E}_{A} d^{n} x+d_{H} \sigma .
\end{aligned}
$$

It follows that the graded derivation

$$
d_{\Lambda}\left(\sum_{0 \leq|\Xi|} \eta\left(G_{R}^{r}\right)^{\Xi} C_{\Xi}^{R}\right) u_{r}^{A, \Lambda} \frac{\partial}{\partial s^{A}}
$$

is a variational symmetry of a Lagrangian $L$ and, consequently, its gauge symmetry parameterized by ghosts $C^{R}$. It factorizes through the gauge symmetry (36) by putting ghosts

$$
c^{r}=\sum_{0 \leq|\Xi|} \eta\left(G_{R}^{r}\right)^{\Xi} C_{\Xi}^{R}
$$

Thus, we come to the following definition.

Definition 9: The odd graded derivation $u$ (36) is said to be a complete non-trivial gauge symmetry of Lagrangian field theory associated to complete non-trivial NI (16).

For instance, if a complete non-trivial gauge symmetry (36) is of second jet order in ghosts, i.e.,

$$
u=\left(c^{r} u_{r}^{A}+c_{\mu}^{r} u_{r}^{A, \mu}+c_{\nu \mu}^{r} u_{r}^{A, \nu \mu}\right) \partial_{A},
$$

the corresponding NI (16) take the form

$$
u_{r}^{A} \mathcal{E}_{A}-d_{\mu}\left(u_{r}^{A, \mu} \mathcal{E}_{A}\right)+d_{\nu \mu}\left(u_{r}^{A, \nu \mu} \mathcal{E}_{A}\right)=0 .
$$


Turn now to the relation (37). For $k=1$, it takes the form

$$
\sum_{0 \leq|\Sigma|} d_{\Sigma} u^{r} \frac{\partial}{\partial c_{\Sigma}^{r}} u^{A}=\bar{\delta}\left(\alpha^{A}\right)
$$

of a first-stage gauge symmetry condition on-shell which the non-trivial gauge symmetry $u$ (36) satisfies. Therefore, one can treat the odd graded derivation

$$
u^{(1)}=u^{r} \frac{\partial}{\partial c^{r}}, \quad u^{r}=\sum_{0 \leq|\Lambda|} c_{\Lambda}^{r_{1}} \eta\left(\Delta_{r_{1}}^{r}\right)^{\Lambda},
$$

as a first-stage gauge symmetry associated to the complete non-trivial first-stage NI

$$
\sum_{0 \leq|\Lambda|} \Delta_{r_{1}}^{r, \Lambda} d_{\Lambda}\left(\sum_{0 \leq|\Sigma|} \Delta_{r}^{A, \Sigma} \bar{s}_{\Sigma A}\right)=-\bar{\delta}\left(\sum_{0 \leq|\Sigma|,|\Xi|} h_{r_{1}}^{(B, \Sigma)(A, \Xi)} \bar{s}_{\Sigma B} \bar{s}_{\Xi A}\right)
$$

Iterating the arguments, one comes to the relation (37) which provides a $k$-stage gauge symmetry condition which is associated to the complete non-trivial $k$-stage NI (23).

Proposition 10: Conversely, given the $k$-stage gauge symmetry condition (37), the $k$-stage NI (23) are reproduced. ${ }^{3}$

Accordingly, we call the odd graded derivation $u_{(k)}(38)$ the $k$-stage gauge symmetry. It is complete as follows. ${ }^{3}$ Let

$$
\sum_{0 \leq|\Xi|} C^{R_{k}} G_{R_{k}}^{r_{k}, \Xi} d_{\Xi} \Delta_{r_{k}} d^{n} x
$$

be a projective $C^{\infty}(X)$-module of finite rank of non-trivial $k$-stage NI (15) factorizing through the complete ones (21), and which are parameterized by the corresponding ghosts $C^{R_{k}}$. One can show that it defines a $k$-stage gauge symmetry factorizing through $u^{(k)}(38)$ by putting $k$-stage ghosts

$$
c^{r_{k}}=\sum_{0 \leq|\Xi|} \eta\left(G_{R_{k}}^{r_{k}}\right)^{\Xi} C_{\Xi}^{R_{k}}
$$

Definition 11: The odd graded derivation $u_{(k)}(38)$ is said to be a complete non-trivial $k$-stage gauge symmetry of a Lagrangian $L$.

In accordance with Definitions 9 and 11, components of the ascent operator $\mathbf{u}(31)$ are complete non-trivial gauge and higher-stage gauge symmetries. Therefore, we agree to call this operator the gauge operator. In these terms, Theorem 7 is the inverse second Noether theorem. The corresponding direct second Noether theorem is stated by Propositions 8 and 10. 


\section{ALGEBRA OF GAUGE SYMMETRIES}

In contrast with the KT operator (20), the gauge operator $\mathbf{u}(30)$ need not be nilpotent. Following the example of Yang-Mills gauge theory, let us study its extension to a nilpotent graded derivation

$$
\begin{array}{r}
\mathbf{b}=\mathbf{u}+\gamma=\mathbf{u}+\sum_{1 \leq k \leq N+1} \gamma^{(k)}=\mathbf{u}+\sum_{1 \leq k \leq N+1} \gamma^{r_{k-1}} \frac{\partial}{\partial c^{r_{k-1}}}= \\
\left(u^{A} \frac{\partial}{\partial s^{A}}+\gamma^{r} \frac{\partial}{\partial c^{r}}\right)+\sum_{0 \leq k \leq N-1}\left(u^{r_{k}} \frac{\partial}{\partial c^{r_{k}}}+\gamma^{r_{k+1}} \frac{\partial}{\partial c^{r_{k+1}}}\right)
\end{array}
$$

of ghost number 1 by means of antifield-free terms $\gamma^{(k)}$ of higher polynomial degree in ghosts $c^{r_{i}}$ and their jets $c_{\Lambda}^{r_{i}}, 0 \leq i<k$. We call $\mathbf{b}(41)$ the BRST operator. The following necessary condition holds.

Proposition 12: The gauge operator (30) admits the nilpotent extension (41) only if the gauge symmetry conditions (37) and the higher-stage NI (23) are satisfied off-shell.

Proof: It is easily justified that, if the graded derivation b (41) is nilpotent, then the right hand sides of the equalities (37) equal zero, i.e.,

$$
u^{(k+1)}\left(u^{(k)}\right)=0, \quad 0 \leq k \leq N-1, \quad u^{(0)}=u .
$$

Using the relations (26) - (28), one can show that, in this case, the right hand sides of the higher-stage NI (23) also equal zero. ${ }^{4}$ It follows that the summand $G_{r_{k}}$ of each cocycle $\Delta_{r_{k}}$ (21) is $\delta_{k-1}$-closed. Then its summand $h_{r_{k}}$ is also $\delta_{k-1}$-closed and, consequently, $\delta_{k-2}$-closed. Hence it is $\delta_{k-1}$-exact by virtue of Condition 6 . Therefore, $\Delta_{r_{k}}$ contains only the term $G_{r_{k}}$ linear in antifields.

It follows at once from the equalities (42) that the gauge operator of higher-stage gauge symmetries

$$
u_{H S}=\mathbf{u}-u=u^{(1)}+\cdots+u^{(N)}
$$

is nilpotent, and $\mathbf{u}(\mathbf{u})=u(\mathbf{u})$. Therefore, the nilpotency condition for the BRST operator b (41) takes the form

$$
\mathbf{b}(\mathbf{b})=(u+\gamma)(\mathbf{u})+\left(u+u_{H S}+\gamma\right)(\gamma)=0 .
$$

Let us denote

$$
\begin{aligned}
& \gamma^{(0)}=0, \quad \gamma^{(k)}=\gamma_{(2)}^{(k)}+\cdots+\gamma_{(k+1)}^{(k)}, \quad k=1, \ldots, N+1, \quad \gamma^{(N+2)}=0, \\
& \gamma_{(i)}^{r_{k-1}}=\sum_{k_{1}+\cdots+k_{i}=k+1-i}\left(\sum_{0 \leq \Lambda_{k_{1}}, \ldots, \Lambda_{k_{i}}} \gamma_{(i) r_{k_{1}}, \ldots, r_{k_{i}}}^{r_{k-1}, \Lambda_{k_{1}}, \ldots, \Lambda_{k_{i}}} c_{\Lambda_{k_{1}}}^{r_{k_{1}}} \cdots c_{\Lambda_{k_{i}}}^{r_{k_{i}}}\right), \quad k=1, \ldots, N+1,
\end{aligned}
$$


where $\gamma_{(i)}^{(k)}$ are terms of polynomial degree $2 \leq i \leq k+1$ in ghosts. Then the nilpotent property (43) of $\mathbf{b}$ falls into a set of equalities

$$
\begin{aligned}
& u^{(k+1)}\left(u^{(k)}\right)=0, \quad 0 \leq k \leq N-1, \\
& \left(u+\gamma_{(2)}^{(k+1)}\right)\left(u^{(k)}\right)+u_{H S}\left(\gamma_{(2)}^{(k)}\right)=0, \quad 0 \leq k \leq N+1, \\
& \gamma_{(i)}^{(k+1)}\left(u^{(k)}\right)+u\left(\gamma_{(i-1)}^{(k)}\right)+u_{H S}\left(\gamma_{(i)}^{k}\right)+ \\
& \quad \sum_{2 \leq m \leq i-1} \gamma_{(m)}\left(\gamma_{(i-m+1)}^{(k)}\right)=0, \quad i-2 \leq k \leq N+1,
\end{aligned}
$$

of ghost polynomial degree 1,2 and $3 \leq i \leq N+3$, respectively.

The equalities (44) are exactly the gauge symmetry conditions (42) in Proposition 12.

The equality (45) for $k=0$ reads

$$
\left(u+\gamma^{(1)}\right)(u)=0, \quad \sum_{0 \leq|\Lambda|}\left(d_{\Lambda}\left(u^{A}\right) \partial_{A}^{\Lambda} u^{B}+d_{\Lambda}\left(\gamma^{r}\right) u_{r}^{B, \Lambda}\right)=0
$$

It takes the form of the Lie antibracket

$$
[u, u]=-2 \gamma^{(1)}(u)=-2 \sum_{0 \leq|\Lambda|} d_{\Lambda}\left(\gamma^{r}\right) u_{r}^{B, \Lambda} \partial_{B}
$$

of the odd gauge symmetry $u$. Its right-hand side is a non-linear differential operator on the module $\mathcal{C}^{(0)}$ of ghosts taking values in the real space $\mathcal{G}_{L}$ of variational symmetries. Following Definition 2 extended to Lagrangian theories of odd fields, we treat it as a generalized gauge symmetry factorizing through the gauge symmetry $u$. Thus, we come to the following.

Proposition 13: The gauge operator (30) admits the nilpotent extension (41) only if the Lie antibracket of the odd gauge symmetry $u(36)$ is a generalized gauge symmetry factorizing through $u$.

The equalities $(45)-(46)$ for $k=1$ take the form

$$
\begin{aligned}
& \left(u+\gamma_{(2)}^{(2)}\right)\left(u^{(1)}\right)+u^{(1)}\left(\gamma^{(1)}\right)=0 \\
& \gamma_{(3)}^{(2)}\left(u^{(1)}\right)+\left(u+\gamma^{(1)}\right)\left(\gamma^{(1)}\right)=0 .
\end{aligned}
$$

In particular, if Lagrangian field theory is irreducible, i.e., $u^{(k)}=0$ and $\mathbf{u}=u$, the BRST operator reads

$$
\mathbf{b}=u+\gamma^{(1)}=u^{A} \partial_{A}+\gamma^{r} \partial_{r}=\sum_{0 \leq|\Lambda|} u_{r}^{A, \Lambda} c_{\Lambda}^{r} \partial_{A}+\sum_{0 \leq|\Lambda|,|\Xi|} \gamma_{p q}^{r, \Lambda, \Xi} c_{\Lambda}^{p} c_{\Xi}^{q} \partial_{r}
$$


and the nilpotency conditions (49) - (50) are reduced to the equality

$$
\left(u+\gamma^{(1)}\right)\left(\gamma^{(1)}\right)=0
$$

Furthermore, let a gauge symmetry $u$ be affine in fields $s^{A}$ and their jets. Then it follows from the nilpotency condition (47) that the BRST term $\gamma^{(1)}$ is independent of original fields and their jets. Then the relation (51) takes the form of the Jacobi identity

$$
\left.\gamma^{(1)}\right)\left(\gamma^{(1)}\right)=0
$$

for coefficient functions $\gamma_{p q}^{r, \Lambda, \Xi}(x)$ in the Lie antibracket (48).

The relations (52) and (48) motivate us to think of the equalities (45) - (46) in a general case of reducible gauge symmetries as being sui generis commutation relations and Jacobi identities, respectively. Moreover, based on Proposition 13, we say that non-trivial gauge symmetries are algebraically closed (in the terminology of Ref. [5]) if the gauge operator $\mathbf{u}$ (31) admits the nilpotent BRST extension b (41).

\section{YANG-MILLS SUPERGAUGE THEORY}

Yang-Mills supergauge theory exemplifies theory of odd fields.

Let $\mathfrak{g}=\mathfrak{g}_{0} \oplus \mathfrak{g}_{1}$ be a finite-dimensional real Lie superalgebra with the basis $\left\{e_{r}\right\}$, $r=1, \ldots, m$, and real structure constants $c_{i j}^{r}$. Recall that

$$
\begin{aligned}
& c_{i j}^{r}=-(-1)^{[i][j]} c_{j i}^{r}, \quad[r]=[i]+[j], \\
& (-1)^{[i][b]} c_{i j}^{r} c_{a b}^{j}+(-1)^{[a][i]} c_{a j}^{r} c_{b i}^{j}+(-1)^{[b][a]} c_{b j}^{r} c_{i a}^{j}=0,
\end{aligned}
$$

where $[r]$ denotes the Grassmann parity of $e_{r}$. Given the universal enveloping algebra $\overline{\mathfrak{g}}$ of $\mathfrak{g}$, we assume that there is an even quadratic Casimir element $h^{i j} e_{i} e_{j}$ of $\overline{\mathfrak{g}}$ such that the matrix $h^{i j}$ is non-degenerate. The Yang-Mills theory on $X=\mathbb{R}^{n}$ associated to this Lie superalgebra is described by the DGA $\mathcal{P}_{\infty}^{*}[F ; Y]$ where

$$
F=\mathfrak{g} \underset{X}{\otimes} T^{*} X, \quad Y=\mathfrak{g}_{0} \underset{X}{\otimes} T^{*} X .
$$

Its local basis is $\left(a_{\lambda}^{r}\right),\left[a_{\lambda}^{r}\right]=[r]$. First jets of its elements admit the canonical splitting

$$
a_{\lambda \mu}^{r}=\frac{1}{2}\left(\mathcal{F}_{\lambda \mu}^{r}+\mathcal{S}_{\lambda \mu}^{r}\right)=\frac{1}{2}\left(a_{\lambda \mu}^{r}-a_{\mu \lambda}^{r}+c_{i j}^{r} a_{\lambda}^{i} a_{\mu}^{j}\right)+\frac{1}{2}\left(a_{\lambda \mu}^{r}+a_{\mu \lambda}^{r}-c_{i j}^{r} a_{\lambda}^{i} a_{\mu}^{j}\right) .
$$


Given a constant metric $g$ on $\mathbb{R}^{n}$, the Yang-Mills Lagrangian reads

$$
L_{Y M}=\frac{1}{4} h_{i j} g^{\lambda \mu} g^{\beta \nu} \mathcal{F}_{\lambda \beta}^{i} \mathcal{F}_{\mu \nu}^{j} d^{n} x .
$$

Its variational derivatives $\mathcal{E}_{r}^{\lambda}$ obey the irreducible NI

$$
c_{j i}^{r} a_{\lambda}^{i} \mathcal{E}_{r}^{\lambda}+d_{\lambda} \mathcal{E}_{j}^{\lambda}=0 .
$$

Therefore, we enlarge the DGA $\mathcal{P}_{\infty}^{*}[F ; Y]$ to the DGA

$$
P_{\infty}^{*}\{0\}=\mathcal{P}_{\infty}^{*}\left[F \underset{Y}{\oplus} E_{0} ; Y\right], \quad E_{0}=X \times\left(\mathfrak{g}_{1} \oplus \mathfrak{g}_{0}\right),
$$

whose local basis $\left(a_{\lambda}^{r}, c^{r}\right),\left[c^{r}\right]=([r]+1) \bmod 2$, contains ghosts $c^{r}$ of ghost number 1 . Then the gauge operator $(31)$ reads

$$
\mathbf{u}=\left(-c_{j i}^{r} c^{j} a_{\lambda}^{i}+c_{\lambda}^{r}\right) \frac{\partial}{\partial a_{\lambda}^{r}} .
$$

It admits the nilpotent BRST extension

$$
\mathbf{b}=\mathbf{u}+\xi=\left(-c_{j i}^{r} c^{j} a_{\lambda}^{i}+c_{\lambda}^{r}\right) \frac{\partial}{\partial a_{\lambda}^{r}}-\frac{1}{2}(-1)^{[i]} c_{i j}^{r} c^{i} c^{j} \frac{\partial}{\partial c^{r}} .
$$

\section{TOPOLOGICAL CHERN-SIMONS THEORY}

We consider gauge theory of principal connections on a principal bundle $P \rightarrow X$ with a structure real Lie group $G$. In contrast with the Yang-Mills Lagrangian, the Chern-Simons (henceforth CS) Lagrangian is independent of a metric on $X$. Therefore, its non-trivial gauge symmetries are wider than those of the Yang-Mills one. Moreover, some of them become trivial if $\operatorname{dim} X=3$.

Note that one usually considers the local CS Lagrangian which is the local CS form derived from the local transgression formula for the Chern characteristic form. The global CS Lagrangian is well defined, but depends on a background gauge potential. ${ }^{16-18}$

The fiber bundle $J^{1} P \rightarrow C$ is a trivial $G$-principal bundle canonically isomorphic to $C \times P \rightarrow C .{ }^{1}$ This bundle admits the canonical principal connection

$$
\mathcal{A}=d x^{\lambda} \otimes\left(\partial_{\lambda}+a_{\lambda}^{p} \varepsilon_{p}\right)+d a_{\lambda}^{r} \otimes \partial_{r}^{\lambda} .
$$


Its curvature defines the canonical $(V P / G)$-valued 2-form

$$
\mathfrak{F}=\left(d a_{\mu}^{r} \wedge d x^{\mu}+\frac{1}{2} c_{p q}^{r} a_{\lambda}^{p} a_{\mu}^{q} d x^{\lambda} \wedge d x^{\mu}\right) \otimes e_{r}
$$

on $C$. Given a section $A$ of $C \rightarrow X$, the pull-back

$$
F_{A}=A^{*} \mathfrak{F}=\frac{1}{2} F_{\lambda \mu}^{r} d x^{\lambda} \wedge d x^{\mu} \otimes e_{r}
$$

of $\mathfrak{F}$ onto $X$ is the strength form of a gauge potential $A$.

Let $I_{k}(\chi)=b_{r_{1} \ldots r_{k}} \chi^{r_{1}} \cdots \chi^{r_{k}}$ be a $G$-invariant polynomial of degree $k>1$ on the Lie algebra $\mathfrak{g}$ of $G$. With $\mathfrak{F}(54)$, one can associate to $I_{k}$ the closed $2 k$-form

$$
P_{2 k}(\mathfrak{F})=b_{r_{1} \ldots r_{k}} \mathfrak{F}^{r_{1}} \wedge \cdots \wedge \mathfrak{F}^{r_{k}}, \quad k \leq \operatorname{dim} X
$$

on $C$ which is invariant under automorphisms of $C$ induced by vertical automorphisms of $P$. Given a section $B$ of $C \rightarrow X$, the pull-back $P_{2 k}\left(F_{B}\right)=B^{*} P_{2 k}(\mathfrak{F})$ of $P_{2 k}(\mathfrak{F})$ is a closed characteristic form on $X$. Let the same symbol stand for its pull-back onto $C$. Since $C \rightarrow X$ is an affine bundle and the de Rham cohomology of $C$ equals that of $X$, the forms $P_{2 k}(\mathfrak{F})$ and $P_{2 k}\left(F_{B}\right)$ possess the same cohomology class $\left[P_{2 k}(\mathfrak{F})\right]=\left[P_{2 k}\left(F_{B}\right)\right]$ for any principal connection $B$. Thus, $I_{k}(\chi) \mapsto\left[P_{2 k}\left(F_{B}\right)\right] \in H_{D R}^{*}(X)$ is the familiar Weil homomorphism. Furthermore, we obtain the transgression formula

$$
P_{2 k}(\mathfrak{F})-P_{2 k}\left(F_{B}\right)=d \mathfrak{S}_{2 k-1}(a, B)
$$

on $C{ }^{16}$ Its pull-back by means of a section $A$ of $C \rightarrow X$ gives the transgression formula

$$
P_{2 k}\left(F_{A}\right)-P_{2 k}\left(F_{B}\right)=d \mathfrak{S}_{2 k-1}(A, B)
$$

on $X$. For instance, if $P_{2 k}(\mathfrak{F})$ is the characteristic Chern $2 k$-form, then $\mathfrak{S}_{2 k-1}(a, B)$ is the CS $(2 k-1)$-form. In particular, one can choose the local section $B=0$. Then, $\mathfrak{S}_{2 k-1}(a, 0)$ is the local CS form. Let $\mathfrak{S}_{2 k-1}(A, 0)$ be its pull-back onto $X$ by means of a section $A$ of $C \rightarrow X$. Then the CS form $\mathfrak{S}_{2 k-1}(a, B)(56)$ admits the decomposition

$$
\mathfrak{S}_{2 k-1}(a, B)=\mathfrak{S}_{2 k-1}(a, 0)-\mathfrak{S}_{2 k-1}(B, 0)+d K_{2 k-1} .
$$

The transgression formula (56) also yields the transgression formula

$$
P_{2 k}(\mathcal{F})-P_{2 k}\left(F_{B}\right)=d_{H}\left(h_{0} \mathfrak{S}_{2 k-1}(a, B)\right),
$$




$$
\begin{aligned}
& h_{0} \mathfrak{S}_{2 k-1}(a, B)=k \int_{0}^{1} \mathcal{P}_{2 k}(t, B) d t \\
& \mathcal{P}_{2 k}(t, B)=b_{r_{1} \ldots r_{k}}\left(a_{\mu_{1}}^{r_{1}}-B_{\mu_{1}}^{r_{1}}\right) d x^{\mu_{1}} \wedge \mathcal{F}^{r_{2}}(t, B) \wedge \cdots \wedge \mathcal{F}^{r_{k}}(t, B), \\
& \mathcal{F}^{r_{j}}(t, B)=\frac{1}{2}\left[t a_{\lambda_{j} \mu_{j}}^{r_{j}}+(1-t) \partial_{\lambda_{j}} B_{\mu_{j}}^{r_{j}}-t a_{\mu_{j} \lambda_{j}}^{r_{j}}-(1-t) \partial_{\mu_{j}} B_{\lambda_{j}}^{r_{j}}+\right. \\
& \quad \frac{1}{2} c_{p q}^{r_{j}}\left(t a_{\lambda_{j}}^{p}+(1-t) B_{\lambda_{j}}^{p}\right)\left(t a_{\mu_{j}}^{q}+(1-t) B_{\mu_{j}}^{q}\right] d x^{\lambda_{j}} \wedge d x^{\mu_{j}} \otimes e_{r},
\end{aligned}
$$

on $J^{1} C$. If $2 k-1=\operatorname{dim} X$, the density $L_{C S}(B)=h_{0} \mathfrak{S}_{2 k-1}(a, B)$ (58) is the global CS Lagrangian of topological CS theory. The decomposition (57) induces the decomposition

$$
L_{C S}(B)=h_{0} \mathfrak{S}_{2 k-1}(a, 0)-\mathfrak{S}_{2 k-1}(B, 0)+d_{H} h_{0} K_{2 k-1}
$$

For instance, if $\operatorname{dim} X=3$, the global CS Lagrangians reads

$$
\begin{aligned}
& L_{C S}(B)=\left[\frac{1}{2} h_{m n} \varepsilon^{\alpha \beta \gamma} a_{\alpha}^{m}\left(\mathcal{F}_{\beta \gamma}^{n}-\frac{1}{3} c_{p q}^{n} a_{\beta}^{p} a_{\gamma}^{q}\right)\right] d^{n} x- \\
& {\left[\frac{1}{2} h_{m n} \varepsilon^{\alpha \beta \gamma} B_{\alpha}^{m}\left(F(B)_{\beta \gamma}^{n}-\frac{1}{3} c_{p q}^{n} B_{\beta}^{p} B_{\gamma}^{q}\right)\right] d^{n} x-d_{\alpha}\left(h_{m n} \varepsilon^{\alpha \beta \gamma} a_{\beta}^{m} B_{\gamma}^{n}\right) d^{n} x,}
\end{aligned}
$$

where $\varepsilon^{\alpha \beta \gamma}$ is the skew-symmetric Levi-Civita tensor.

Since the density $-\mathfrak{S}_{2 k-1}(B, 0)+d_{H} h_{0} K_{2 k-1}$ is variationally trivial, the global CS Lagrangian (59) possesses the same NI and gauge symmetries as the local one $L_{C S}=$ $h_{0} \mathfrak{S}_{2 k-1}(a, 0)$. They are the following.

Infinitesimal generators of local one-parameter groups of automorphisms of a principal bundle $P$ are $G$-invariant projectable vector fields $v_{P}$ on $P$. They are identified with sections

$$
v_{P}=\tau^{\lambda} \partial_{\lambda}+\xi^{r} e_{r}
$$

of the vector bundle $T_{G} P=T P / G \rightarrow X$, and yield vector fields

$$
v_{C}=\tau^{\lambda} \partial_{\lambda}+\left(-c_{p q}^{r} \xi^{p} a_{\lambda}^{q}+\partial_{\lambda} \xi^{r}-a_{\mu}^{r} \partial_{\lambda} \tau^{\mu}\right) \partial_{r}^{\lambda}
$$

on the bundle of principal connections $C .{ }^{1}$ Sections $v_{P}(61)$ play a role of gauge parameters. One can show that vector fields (62) are variational symmetries of the global CS Lagrangian $L_{C S}(B)$. By virtue of Proposition 5, the vertical part

$$
v_{V}=\left(-c_{p q}^{r} \xi^{p} a_{\lambda}^{q}+\partial_{\lambda} \xi^{r}-a_{\mu}^{r} \partial_{\lambda} \tau^{\mu}-\tau^{\mu} a_{\mu \lambda}^{r}\right) \partial_{r}^{\lambda}
$$

of a vector field $v_{C}(62)$ is also a variational symmetry of $L_{C S}(B)$. 
Let us consider the DGA $\mathcal{P}_{\infty}^{*}\left[T_{G} P ; C\right]$ possessing the local basis $\left(a_{\lambda}^{r}, c^{\lambda}, c^{r}\right)$ of even fields $a_{\lambda}^{r}$ and odd ghosts $c^{\lambda}, c^{r}$. Substituting these ghosts for gauge parameters in the vector field $v_{V}(63)$, we obtain the odd vertical graded derivation

$$
u=\left(-c_{p q}^{r} c^{p} a_{\lambda}^{q}+c_{\lambda}^{r}-c_{\lambda}^{\mu} a_{\mu}^{r}-c^{\mu} a_{\mu \lambda}^{r}\right) \partial_{r}^{\lambda}
$$

of the DGA $\mathcal{P}_{\infty}^{*}\left[T_{G} P ; C\right]$. This graded derivation as like as vector fields $v_{V}(63)$ is a variational and, consequently, gauge symmetry of the CS Lagrangian $L_{C S}(B)$. By virtue of the formulas (39) - (40), the corresponding NI read

$$
\bar{\delta} \Delta_{j}=-c_{j i}^{r} a_{\lambda}^{i} \mathcal{E}_{r}^{\lambda}-d_{\lambda} \mathcal{E}_{j}^{\lambda}=0, \quad \bar{\delta} \Delta_{\mu}=-a_{\mu \lambda}^{r} \mathcal{E}_{r}^{\lambda}+d_{\lambda}\left(a_{\mu}^{r} \mathcal{E}_{r}^{\lambda}\right)=0
$$

They are irreducible non-trivial, unless $\operatorname{dim} X=3$. Therefore, the gauge operator (30) is $\mathbf{u}=u$. It admits the nilpotent BRST extension

$$
\mathbf{b}=\left(-c_{j i}^{r} c^{j} a_{\lambda}^{i}+c_{\lambda}^{r}-c_{\lambda}^{\mu} a_{\mu}^{r}-c^{\mu} a_{\mu \lambda}^{r}\right) \frac{\partial}{\partial a_{\lambda}^{r}}-\frac{1}{2} c_{i j}^{r} c^{i} c^{j} \frac{\partial}{\partial c^{r}}+c_{\mu}^{\lambda} c^{\mu} \frac{\partial}{\partial c^{\lambda}} .
$$

If $\operatorname{dim} X=3$, the CS Lagrangian takes the form (60), and the corresponding EulerLagrange operator reads

$$
\delta L_{C S}(B)=\mathcal{E}_{r}^{\lambda} \theta_{\lambda}^{r} \wedge d^{n} x, \quad \mathcal{E}_{r}^{\lambda}=h_{r p} \varepsilon^{\lambda \beta \gamma} \mathcal{F}_{\beta \gamma}^{p} .
$$

A glance at the NI (65) shows that they are equivalent to NI

$$
\bar{\delta} \Delta_{j}=-c_{j i}^{r} a_{\lambda}^{i} \mathcal{E}_{r}^{\lambda}-d_{\lambda} \mathcal{E}_{j}^{\lambda}=0, \quad \bar{\delta} \Delta_{\mu}^{\prime}=\bar{\delta} \Delta_{\mu}+a_{\mu}^{r} \bar{\delta} \Delta_{r}=c^{\mu} \mathcal{F}_{\lambda \mu}^{r} \mathcal{E}_{r}^{\lambda}=0
$$

These NI define the gauge symmetry $u(64)$ written in the form

$$
u=\left(-c_{p q}^{r} c^{\prime p} a_{\lambda}^{q}+c_{\lambda}^{\prime r}+c^{\mu} \mathcal{F}_{\lambda \mu}^{r}\right) \partial_{r}^{\lambda}
$$

where $c^{\prime r}=c^{r}-a_{\mu}^{r} c^{\mu}$. It is readily observed that, if $\operatorname{dim} X=3$, the $\mathrm{NI} \bar{\delta} \Delta_{\mu}^{\prime}$ (66) are trivial. Then the corresponding part $\left.c^{\mu} \mathcal{F}_{\lambda \mu}^{r}\right) \partial_{r}^{\lambda}$ of the gauge symmetry $u(67)$ is also trivial. Consequently, the non-trivial gauge symmetry of the CS Lagrangian (60) is

$$
u=\left(-c_{p q}^{r} c^{\prime p} a_{\lambda}^{q}+c_{\lambda}^{\prime r}\right) \partial_{r}^{\lambda}
$$

\section{GAUGE GRAVITATION THEORY}


Gravitation theory can be formulated as gauge theory on natural bundles $T$ over an oriented four-dimensional manifold $X .^{19,20}$ It is metric-affine gravitation theory whose Lagrangian $L_{M A}$ is invariant under general covariant transformations. Infinitesimal generators of local one-parameter groups of these transformations are the functorial lift (i.e., the Lie algebra monomorphism) of vector fields on $X$ onto a natural bundle. Vector fields on $X$ are gauge parameters of general covariant transformations. Natural bundles are exemplified by tensor bundles over $X$. The principal bundle $L X$ of linear frames in the tangent bundle $T X$ of $X$ and associated tensor bundles exemplify natural bundle.

Dynamic variables of gauge gravitation theory on natural bundles are linear connections and pseudo-Riemannian metrics on $X$. Linear connections on $X$ are principal connections on the principal frame bundle $L X$ with the structure group $G L_{4}=G L^{+}(4, \mathbb{R})$. They are represented by global sections of the quotient bundle $C_{K}=J^{1} L X / G L_{4}$. It is also a natural bundle provided with bundle coordinates $\left(x^{\lambda}, k_{\lambda}{ }^{\nu}{ }_{\alpha}\right)$ such that components $k_{\lambda}{ }^{\nu}{ }_{\alpha} \circ K=K_{\lambda{ }^{\nu}}$ of a section $K$ of $C_{K} \rightarrow X$ are coefficient of the linear connection

$$
K=d x^{\lambda} \otimes\left(\partial_{\lambda}+K_{\lambda}{ }^{\mu}{ }_{\nu} \dot{x}^{\nu} \dot{\partial}_{\mu}\right)
$$

on $T X$ with respect to the holonomic bundle coordinates $\left(x^{\lambda}, \dot{x}^{\lambda}\right)$. The first order jet manifold $J^{1} C_{K}$ of $C_{K}$ admits the canonical decomposition taking the coordinate form

$$
\begin{gathered}
k_{\lambda \mu}{ }^{\alpha}{ }_{\beta}=\frac{1}{2}\left(R_{\lambda \mu}{ }^{\alpha}{ }_{\beta}+S_{\lambda \mu}{ }^{\alpha}{ }_{\beta}\right)=\frac{1}{2}\left(k_{\lambda \mu}{ }^{\alpha}{ }_{\beta}-k_{\mu \lambda}{ }_{\beta}{ }_{\beta}+k_{\mu}{ }^{\alpha}{ }_{\varepsilon} k_{\lambda}{ }^{\varepsilon}{ }_{\beta}-k_{\lambda}{ }^{\alpha}{ }_{\varepsilon} k_{\mu}{ }^{\varepsilon} \beta\right)+ \\
\frac{1}{2}\left(k_{\lambda \mu}{ }^{\alpha}{ }_{\beta}+k_{\mu \lambda}{ }^{\alpha}{ }_{\beta}-k_{\mu}{ }^{\alpha}{ }_{\varepsilon} k_{\lambda}{ }^{\varepsilon}{ }_{\beta}+k_{\lambda}{ }^{\alpha}{ }_{\varepsilon} k_{\mu}{ }^{\varepsilon}{ }_{\beta}\right) .
\end{gathered}
$$

If $K$ is a section of $C_{K} \rightarrow X$, then $R \circ K$ is the curvature of a linear connection $K$.

In gravitation theory, the linear frame bundle $L X$ is assumed to admit a Lorentz structure, i.e., reduced principal subbundles with the structure Lorentz group $S O(1,3)$. By virtue of the well-known theorem, there is one-to-one correspondence between these subbundles and the global sections of the quotient bundle $\Sigma=L X / S O(1,3)$. Its sections are pseudo-Riemannian metrics on $X$. Being an open subbundle of the tensor bundle $\vee^{2} T X$, the bundle $\Sigma$ is provided with bundle coordinates $\left(x^{\lambda}, \sigma^{\mu \nu}\right)$.

The total configuration space of gauge gravitation theory is the bundle product $\Sigma \times C_{K}$ coordinated by $\left(x^{\lambda}, \sigma^{\alpha \beta}, k_{\mu}{ }^{\alpha}\right)$. This is a natural bundle admitting the functorial lift

$$
\begin{gathered}
\widetilde{\tau}_{\Sigma K}=\tau^{\mu} \partial_{\mu}+\left(\sigma^{\nu \beta} \partial_{\nu} \tau^{\alpha}+\sigma^{\alpha \nu} \partial_{\nu} \tau^{\beta}\right) \frac{\partial}{\partial \sigma^{\alpha \beta}}+ \\
\left(\partial_{\nu} \tau^{\alpha} k_{\mu}{ }^{\nu}{ }_{\beta}-\partial_{\beta} \tau^{\nu} k_{\mu}{ }^{\alpha}{ }_{\nu}-\partial_{\mu} \tau^{\nu} k_{\nu}{ }^{\alpha}{ }_{\beta}+\partial_{\mu \beta} \tau^{\alpha}\right) \frac{\partial}{\partial k_{\mu}{ }^{\alpha}{ }_{\beta}}
\end{gathered}
$$


of vector fields $\tau=\tau^{\mu} \partial_{\mu}$ on $X{ }^{1,13}$ Let us consider the DGA $\mathcal{S}_{\infty}^{*}\left[\Sigma \times C_{K}\right]$ possessing the local basis $\left(\sigma^{\alpha \beta}, k_{\mu}{ }^{\alpha} \beta\right)$, and let us enlarge it to the DGA

$$
\mathcal{P}_{\infty}^{*}\left[T X ; \Sigma \times C_{K}\right]
$$

possessing the local basis $\left(\sigma^{\alpha \beta}, k_{\mu}{ }^{\alpha}{ }_{\beta}, c^{\mu}\right)$ of even fields $\left(\sigma^{\alpha \beta}, k_{\mu}{ }_{\beta}{ }_{\beta}\right)$ and odd ghosts $\left(c^{\mu}\right)$. Taking the vertical part of vector fields $\widetilde{\tau}_{K \Sigma}(68)$ and replacing gauge parameters $\tau^{\lambda}$ with ghosts $c^{\lambda}$, we obtain the odd vertical graded derivation

$$
\begin{aligned}
u= & u^{\alpha \beta} \frac{\partial}{\partial \sigma^{\alpha \beta}}+u_{\mu}{ }^{\alpha}{ }^{\alpha} \frac{\partial}{\partial k_{\mu}{ }^{\alpha} \beta}=\left(\sigma^{\nu \beta} c_{\nu}^{\alpha}+\sigma^{\alpha \nu} c_{\nu}^{\beta}-c^{\lambda} \sigma_{\lambda}^{\alpha \beta}\right) \frac{\partial}{\partial \sigma^{\alpha \beta}}+ \\
& \left(c_{\nu}^{\alpha} k_{\mu}{ }^{\nu}{ }_{\beta}-c_{\beta}^{\nu} k_{\mu}{ }^{\alpha}{ }_{\nu}-c_{\mu}^{\nu} k_{\nu}{ }^{\alpha}{ }_{\beta}+c_{\mu \beta}^{\alpha}-c^{\lambda} k_{\lambda \mu}{ }^{\alpha}{ }_{\beta}\right) \frac{\partial}{\partial k_{\mu}{ }^{\alpha}{ }_{\beta}}
\end{aligned}
$$

of the DGA (69). It is a gauge symmetry of a gravitation Lagrangian $L_{M A}$. Then by virtue of the formulas (39) - (40), the Euler-Lagrange operator

$$
\left(\mathcal{E}_{\alpha \beta} d \sigma^{\alpha \beta}+\mathcal{E}^{\mu}{ }_{\alpha}{ }^{\beta} d k_{\mu}{ }^{\alpha}{ }\right) \wedge d^{4} x
$$

of this Lagrangian obeys the NI

$$
\begin{aligned}
& -\sigma_{\lambda}^{\alpha \beta} \mathcal{E}_{\alpha \beta}-2 d_{\mu}\left(\sigma^{\mu \beta} \mathcal{E}_{\lambda \beta}-k_{\lambda \mu}{ }^{\alpha}{ }_{\beta} \mathcal{E}^{\mu}{ }_{\alpha}{ }^{\beta}-\right. \\
& \quad d_{\mu}\left[\left(k_{\nu}{ }^{\mu}{ }_{\beta} \delta_{\lambda}^{\alpha}-k_{\nu}{ }^{\alpha}{ }_{\lambda} \delta_{\beta}^{\mu}-k_{\lambda}{ }^{\alpha}{ }_{\beta} \delta_{\nu}^{\mu}\right) \mathcal{E}^{\nu}{ }_{\alpha}{ }^{\beta}\right]+d_{\mu \beta} \mathcal{E}^{\mu}{ }_{\lambda}{ }^{\beta}=0 .
\end{aligned}
$$

These NI are irreducible. Therefore, the gauge operator (30) is $\mathbf{u}=u$. Its BRST extension reads $^{13}$

$$
\mathbf{b}=u+c_{\mu}^{\lambda} c^{\mu} \frac{\partial}{\partial c^{\lambda}}
$$

Note that this BRST operator differs from that in Ref. [21], where metric-affine gravitation theory is treated as gauge theory of the Poincaré group. ${ }^{22-24}$

\section{TOPOLOGICAL BF THEORY}

We address the topological BF theory of two exterior forms $A$ and $B$ of form degree $|A|+$ $|B|=\operatorname{dim} X-1$ on a smooth manifold $X{ }^{25}$ It is reducible degenerate Lagrangian theory which satisfies the homology regularity condition. ${ }^{3,4}$ Its dynamic variables are exterior forms $A$ and $B$ of form degree $|A|+|B|=n-1$ on a manifold $X$. They are sections of the bundle

$$
Y=\stackrel{p}{\wedge} T^{*} X \oplus \stackrel{q}{\wedge} T^{*} X, \quad p+q=n-1,
$$


coordinated by $\left(x^{\lambda}, A_{\mu_{1} \ldots \mu_{p}}, B_{\nu_{1} \ldots \nu_{q}}\right)$. Without a loss of generality, let $q$ be even and $q \geq p$. The corresponding DGA is $\mathcal{O}_{\infty}^{*} Y$. There are the canonical $p$ - and $q$-forms

$$
A=\frac{1}{p !} A_{\mu_{1} \ldots \mu_{p}} d x^{\mu_{1}} \wedge \cdots \wedge d x^{\mu_{p}}, \quad B=\frac{1}{q !} B_{\nu_{p+1} \ldots \nu_{q}} d x^{\nu_{p+1}} \wedge \cdots \wedge d x^{\nu_{p}}
$$

on $Y$. A Lagrangian of the topological BF theory reads

$$
L_{\mathrm{BF}}=A \wedge d_{H} B
$$

Its Euler-Lagrange equations $d_{H} A=0, d_{H} B=0$ obey the NI

$$
d_{H} d_{H} A=0, \quad d_{H} d_{H} B=0 .
$$

Given the vector bundles

$$
\begin{aligned}
& E_{k}=\stackrel{p-k-1}{\wedge} T^{*} X \underset{X}{ } \wedge^{q-k-1} T^{*} X, \quad 0 \leq k<p-1, \\
& E_{k}=\mathbb{R} \underset{X}{\times} \wedge^{q-p} T^{*} X, \quad k=p-1, \\
& E_{k}=\stackrel{q-k-1}{\wedge} T^{*} X, \quad p-1<k<q-1, \\
& E_{q-1}=X \times \mathbb{R},
\end{aligned}
$$

let us consider the DGA $P_{\infty}^{*}\{q-1\}$ with the local basis

$$
\begin{aligned}
& \left\{A_{\mu_{1} \ldots \mu_{p}}, B_{\nu_{p+1} \ldots \nu_{q}}, \varepsilon_{\mu_{2} \ldots \mu_{p}}, \ldots, \varepsilon_{\mu_{p}}, \varepsilon, \xi_{\nu_{p+2} \ldots \nu_{q}}, \ldots, \xi_{\nu_{q}}, \xi\right. \\
& \left.\bar{A}^{\mu_{1} \ldots \mu_{p}}, \bar{B}^{\nu_{p+1} \ldots \nu_{q}}, \bar{\varepsilon}^{\mu_{2} \ldots \mu_{p}}, \ldots, \bar{\varepsilon}^{\mu_{p}}, \bar{\varepsilon}, \bar{\xi}^{\nu_{p+2} \ldots \nu_{q}}, \ldots, \bar{\xi}^{\nu_{q}}, \bar{\xi}\right\} .
\end{aligned}
$$

Then the gauge operator (31) reads

$$
\begin{aligned}
\mathbf{u}= & d_{\mu_{1}} \varepsilon_{\mu_{2} \ldots \mu_{p}} \frac{\partial}{\partial A_{\mu_{1} \mu_{2} \ldots \mu_{p}}}+d_{\nu_{p+1}} \xi_{\nu_{p+2} \ldots \nu_{q}} \frac{\partial}{\partial B_{\nu_{p+1} \nu_{p+2} \ldots \nu_{q}}}+\left[d_{\mu_{2}} \varepsilon_{\mu_{3} \ldots \mu_{p}} \frac{\partial}{\partial \varepsilon_{\mu_{2} \mu_{3} \ldots \mu_{p}}}+\cdots\right. \\
& \left.+d_{\mu_{p}} \varepsilon \frac{\partial}{\partial \varepsilon^{\mu_{p}}}\right]+\left[d_{\nu_{p+2}} \xi_{\nu_{p+3} \ldots \nu_{q}} \frac{\partial}{\partial \xi_{\nu_{p+2} \nu_{p+3} \ldots \nu_{q}}}+\cdots+d_{\nu_{q}} \xi \frac{\partial}{\partial \xi^{\nu_{q}}}\right] .
\end{aligned}
$$

This operator is obviously nilpotent and, thus, is the BRST operator $\mathbf{b}=\mathbf{u}$.

1 L. Mangiarotti, and G. Sardanashvily, Connections in Classical and Quantum Field Theory (World Scientific, Singapore, 2000). 
2 G. Giachetta, L. Mangiarotti, and G. Sardanashvily, Commun. Math. Phys. 259, 103 (2005).

3 D. Bashkirov, G. Giachetta, L. Mangiarotti, and G. Sardanashvily, J. Phys. A 38, 5329 (2005).

4 D. Bashkirov, G. Giachetta, L. Mangiarotti, and G. Sardanashvily, J. Math. Phys. 46, $053517(2005)$.

5 J. Gomis, J. París, and S. Samuel, Phys. Rep. 295, 1 (1995).

6 G. Barnich, F. Brandt, and M. Henneaux, Phys. Rep. 338, 439 (2000).

7 A.Fuster, M.Henneaux and A.Maas, Int. J. Geom. Methods Mod. Phys. 2, 939 (2005).

8 D. Bashkirov, G. Giachetta, L. Mangiarotti, and G. Sardanashvily, Lett. Math. Phys. 83, 237 (2008).

9 R. Fulp, T. Lada, and J. Stasheff, Commun. Math. Phys. 231, 25 (2002).

10 D. Bashkirov and G. Sardanashvily, Int. J. Geom. Methods Mod. Phys. 2, 203 (2005).

11 D. Bashkirov, G. Giachetta, L. Mangiarotti, and G. Sardanashvily, J. Math. Phys. 46, $103513(2005)$.

12 G. Sardanashvily, Int. J. Geom. Methods Mod. Phys. 4, 1335 (2007).

13 G. Sardanashvily, Int. J. Geom. Methods Mod. Phys. 2, 873 (2005).

14 J. Fisch, and M. Henneaux, Commun. Math. Phys. 128, 627 (1990).

15 R. Fulp, T. Lada, and J. Stasheff, Rend. Circ. Mat. Palermo (2) Suppl., No. 71, 115 (2003).

16 G. Giachetta, L. Mangiarotti and G. Sardanashvily, Mod. Phys. Lett. A 18, 2645 (2003).

17 A.Borowoiec, L.Fatibene, M.Ferraris and M.Francaviglia, Int. J. Geom. Methods Mod. Phys. 3, 755 (2006).

18 A.Borowoiec, L.Fatibene, M.Ferraris and S.Mercadante, Int. J. Geom. Methods mod. Phys. 4, 277 (2007). 
19 G. Sardanashvily, Theor. Math. Phys. 132, 1163 (2002).

${ }^{20}$ G. Sardanashvily, Int. J. Geom. Methods Mod. Phys. 3, No.1, v-xx (2006).

${ }^{21}$ F. Gronwald, Phys. Rev. D 57, 961 (1998).

${ }^{22}$ F.Hehl, J.McCrea, E.Mielke and Y.Ne'eman, Phys. Rep. 258, 1 (1995).

23 J.Klauder, Int. J. Geom. Methods Mod. Phys. 3, 81 (2006).

24 Yu.Obukhov, Int. J. Geom. Methods Mod. Phys. 3, 95 (2006).

25 B. Birmingham, M. Blau, M. Rakowski and G. Thompson, Phys. Rep. 209, 129 (1991). 